\title{
Virtual reality exercise to help COVID patients with refractory breathlessness
}

Betka Sophie*1, Kannape Oliver Alan ${ }^{\star 1,2}$, Fasola Jemina ${ }^{1}$, Lance Florian ${ }^{3}$, Cardin Sylvain², Schmid Aline ${ }^{3}$, Similowski Thomas ${ }^{4,5}$, Soccal Paola Marina ${ }^{3}$, Herbelin Bruno**1, Adler Dan**3, Blanke Olaf**1

* joint first authors

** joint last authors

${ }^{1}$ Laboratory of Cognitive Neuroscience, Brain Mind Institute and Center for Neuroprosthetics, Faculty of Life Sciences, Swiss Federal Institute of Technology, (EPFL), 1202, Geneva, Switzerland

${ }^{2}$ MindMaze SA, 1006 Lausanne, Switzerland

${ }^{3}$ Division of Lung Diseases, University Hospital and Geneva Medical School, University of Geneva, Switzerland

${ }^{4}$ Sorbonne Université, INSERM, UMRS1158 Neurophysiologie Respiratoire Expérimentale et Clinique, F-75005 Paris, France

${ }^{5}$ AP-HP, Groupe Hospitalier Universitaire APHP-Sorbonne Université, site Pitié-Salpêtrière, Département R3S (Respiration, Réanimation, Réhabilitation respiratoire, Sommeil), F-75013 Paris, France

Corresponding author: Prof. Olaf Blanke, Dr Dan Adler

Email: olaf.blanke@epfl.ch; Dan.Adler@hcuge.ch

Tel: +41216939621 
medRxiv preprint doi: https://doi.org/10.1101/2021.10.26.21265510; this version posted October 26, 2021. The copyright holder for this preprint (which was not certified by peer review) is the author/funder, who has granted medRxiv a license to display the preprint in

All rights reserved. No reuse allowed without permission.

\section{Summary (maximum length $\mathbf{3 0 0}$ words)}

\section{Background}

Immersive virtual reality (iVR)-based digital therapeutics (DTx) are gaining clinical attention in the field of pain management. Based on known analogy between chronic pain and dyspnea, we investigated the effects of visual respiratory feedback in iVR, on refractory breathlessness in patients recovering from severe COVID-19 pneumonia.

\section{Methods}

We performed a controlled, randomized, single-blind, cross-over clinical study to evaluate an iVR-based intervention to alleviate refractory breathlessness in patients recovering from COVID-19 pneumonia. The single-site study was conducted at the university hospital of Geneva, Switzerland. Patients reported refractory breathlessness ( $\geq 5$ on a 10-point dyspnea scale) and had a MoCA score of $\geq 24$. Cross-over groups were randomly assigned, concealed from the referring clinician. Participants received synchronous (intervention) or asynchronous (control) feedback of their breathing, embodied via a gender-matched avatar in iVR. Prior to the first exposure and following both experimental conditions, patients completed questionnaires. Breathing patterns were captured continuously. The COVVR clinical study is registered with ClinicalTrials.gov (NCT04844567) and is now closed.

\section{Findings}

Study enrollment was open between November 2020 and April 2021. A total of 26 patients (27\% women; age: mean $=57, S D \pm 12$ ) were enrolled; 14 patients were randomly assigned to the "synchronous/asynchronous" sequence, 12 to the "asynchronous/synchronous" sequence. Data was available for all except two $(7.7 \%)$ of 26 patients. The mean rating of breathing comfort was 0.1 at baseline, $0.8 \pm 1.8$ for asynchronous, and $1.3 \pm 1.4$ synchronous feedback (estimated difference of $0.5(95 \% \mathrm{Cl} 0.05$ to $1.04 ; \mathrm{p}<0.05)$ between iVR conditions). Of all patients, $91.2 \%$ were satisfied with the intervention $(1.8 \pm 1.6, t=5.201, p<0.0001,95 \% \mathrm{Cl}$ 1.173 to inf) and $66.7 \%$ perceived it as beneficial for their breathing $(0.7 \pm 1.9, t=1.806, p<0.05$, $95 \% \mathrm{Cl} 0.036$ to inf). No adverse events were reported.

\section{Interpretation}

Based on these findings, we propose that our iVR-based DTx is a feasible and safe neurorehabilitation tool that improves breathing comfort in patients recovering from severe COVID19 infection. More research is needed to generalize this tool in other groups of patients suffering from refractory breathlessness. 
medRxiv preprint doi: https://doi.org/10.1101/2021.10.26.21265510; this version posted October 26, 2021. The copyright holder for this preprint (which was not certified by peer review) is the author/funder, who has granted medRxiv a license to display the preprint in

All rights reserved. No reuse allowed without permission.

Funding

Marie Skłodowska-Curie Individual Fellowship (H2020-MSCA-IF-2019 894111/ RESPVR), Bertarelli Foundation 
medRxiv preprint doi: https://doi.org/10.1101/2021.10.26.21265510; this version posted October 26, 2021. The copyright holder for this preprint (which was not certified by peer review) is the author/funder, who has granted medRxiv a license to display the preprint in

All rights reserved. No reuse allowed without permission.

\section{Introduction}

Chronic breathlessness syndrome has recently been delineated as a disabling sensation of difficult breathing despite optimized treatment of underlying medical conditions ${ }^{1}$. Although chronic breathlessness, as a syndrome, has originally been described in chronic medical conditions, refractory breathlessness (RB) despite optimized treatment, can also be observed in acute settings ${ }^{2}$. The condition has a non-negligible deleterious impact on patients' quality of life and autonomy, by impacting cognition, locomotion, or mental health ${ }^{3,4}$. RB's definition emphasizes the importance of a complete work-up and identification of the underlying condition for the appropriate pathophysiological treatment; it also underlines the current failure to study an under-recognized respiratory suffering ${ }^{1,2}$ and the need for development of evidenced-based interventions for RB. Indeed, breathlessness remains under-recognized in the hospital setting, although it can be easily collected and rated by trained staff and is associated with short and longer-term mortality ${ }^{5}$. Neuroimaging evidence suggests that the perception of breathlessness is underpinned by a neural network involving the insula, dorsal anterior cingulate cortex, amygdala, and medial thalamus and shares important pathways with other brain functions such as pain processing ${ }^{6}$ and bodily self-consciousness ${ }^{8}$. Prior interventions using immersive virtual reality (iVR)-based Digital Therapeutics (DTx), also referred to as digiceuticals ${ }^{9}$, have demonstrated alleviation of chronic pain in patients with complex regional pain syndrome or spinal cord injury ${ }^{10,11}$. In the respiratory domain, visuorespiratory stimulation (based on visuo-respiratory feedback) has been associated with an increased feeling of breathing control (breathing agency) ${ }^{12}$, a reduced negative emotional state related to experimental dyspnea ${ }^{13}$, as well as changes in physiological measures of breathing 7,14 .

Long COVID is a complex condition with multiples symptoms occurring beyond the initial period of COVID-19 infection recovery and affects patients who were managed in the community or in the acute care setting ${ }^{8}$. Like general weakness, malaise, fatigue, and concentration impairments, breathlessness has consistently been reported in long COVID cohorts with a high prevalence of around $25 \%$ (Cl95 18\% to 34\% $)^{7}$. As the subjective experience of breathlessness is poorly associated to specific physiological markers such as pulmonary function tests or lung imaging in long COVID ${ }^{8}$, treatment is challenging and is most often focused on pulmonary rehabilitation ${ }^{17}$. This also implies that a cognitive intervention using a neuro-rehabilitation approach could be tested to understand and alleviate this debilitating symptom.

The present COVid-19 Virtual Reality (COVVR) clinical study was performed to determine the effects of an iVR-based DTx on breathing comfort in patients recovering from severe COVID- 
medRxiv preprint doi: https://doi.org/10.1101/2021.10.26.21265510; this version posted October 26, 2021. The copyright holder for this preprint (which was not certified by peer review) is the author/funder, who has granted medRxiv a license to display the preprint in

All rights reserved. No reuse allowed without permission.

19 pneumonia. We also quantified patients' subjective benefit related to such intervention and the feasibility of using this new breathing rehabilitation tool at home or in the wards in this specific population. 


\section{Method}

\section{Study Design}

A controlled, randomized, single-blind, cross-over clinical study was conducted to evaluate both efficacy and feasibility of a Virtual Reality biofeedback intervention to alleviate RB in patients recovering from severe COVID-19 pneumonia. This single-site study was carried out at the University Hospital (HUG) in Geneva, Switzerland and was approved by the Commission Cantonale d'Ethique de la Recherche de la République et Canton de Genève (2019-02360).

\section{Patients}

Thirty-one patients were recruited for the study (see Figure 1). Clinical inclusion criteria were that patients i) were recovering from severe COVID-19 pneumonia, and ii) presented with RB with a self-rated intensity of five or higher (out of ten) on a visual analogic dyspnea scale. Furthermore, patients had to be able to give consent and to understand and speak French or English. Patients that presented with unstable respiratory, neurological, or cardiac conditions, or psychiatric illness were excluded. Patients that scored below 25 on the Montreal Cognitive Assessment (MoCA) were also excluded.

\section{Randomisation and masking}

As the study employed a crossover design, patients were allocated to one of two starting conditions (synchronous, asynchronous) using a randomization script, prior to data collection. Randomization was not restricted; no stratification or minimization factors were applied. Allocation was concealed to the clinicians screening patients.

Participant masking (blinding) was achieved by keeping both the procedure and the virtual environment identical for both tested conditions. Participants were naïve to the difference in the two conditions which consisted only of a change in feedback synchrony between respiratory movements and avatar luminance. Experimenters were not blinded, as they set up the VR device. However, the instructions were only given to the patient once before wearing the HMD and starting the intervention and applied to both conditions. 


\section{Procedure}

\section{Screening}

Patients were screened by a respiratory physician during morning rounds. Individuals were referred to the study. It was then verified that the patients met the inclusion criteria by performing an anamnestic interview as well as the MoCA cognitive assessment.

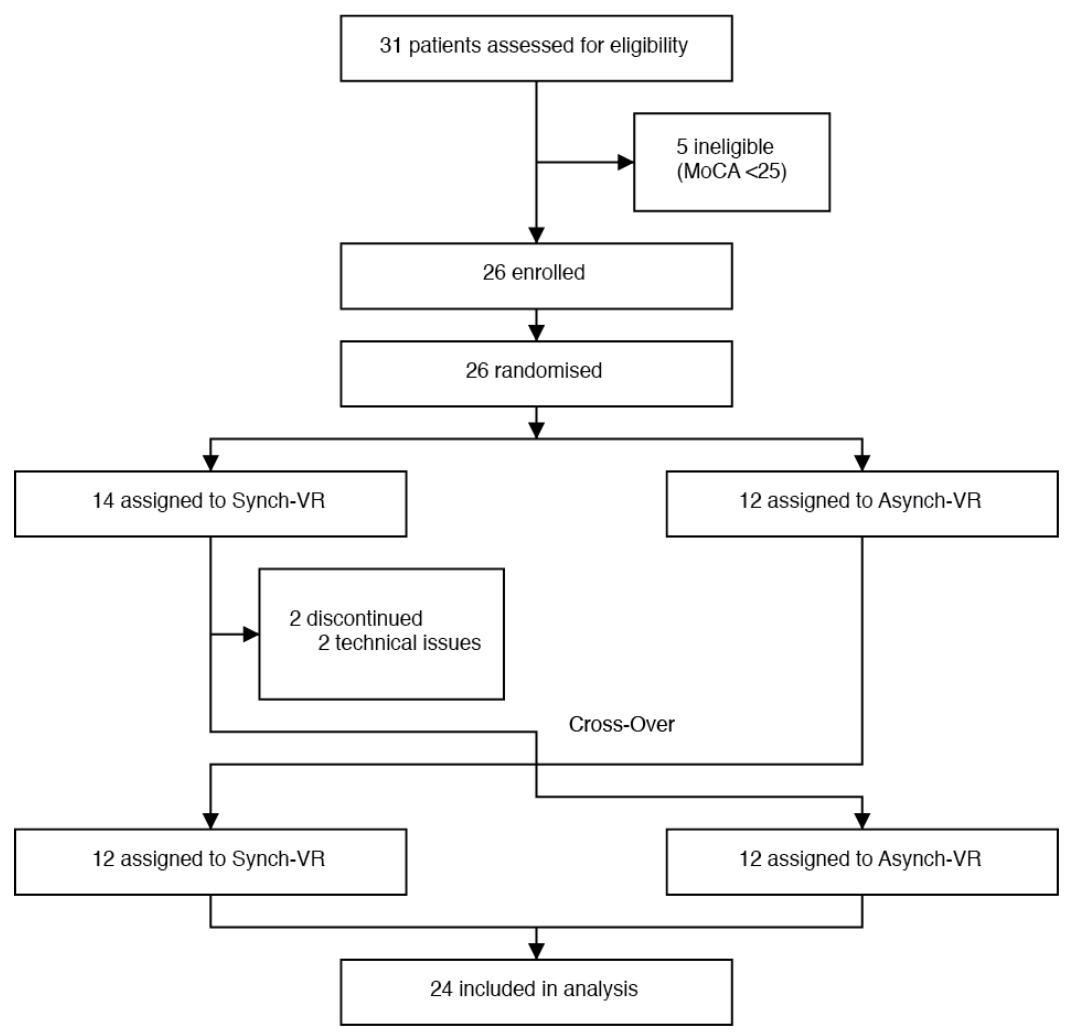

Figure 1 : Study profile

Setup

Eligible patients were installed in a semi-seated position in their hospital bed and wore a beltmounted linear force sensor (Go Direct® Respiration belt, Vernier, Beaverton (OR), USA) fitted on the abdomen to allow proper recording of respiratory movements. They were also equipped with a head-mounted display (Zeiss VR ONEPLUS, Oberkochen, Germany) that holds a VR compatible smartphone (Samsung Galaxy S8, Seoul, South Korea). The smartphone ran the VR simulation and connected via Bluetooth ${ }^{\circledR}$ to the respiration belt. Software developed by the laboratory in collaboration with MindMaze allowed to collect and filter respiratory data and to render a computer-generated virtual environment in real-time. 
A

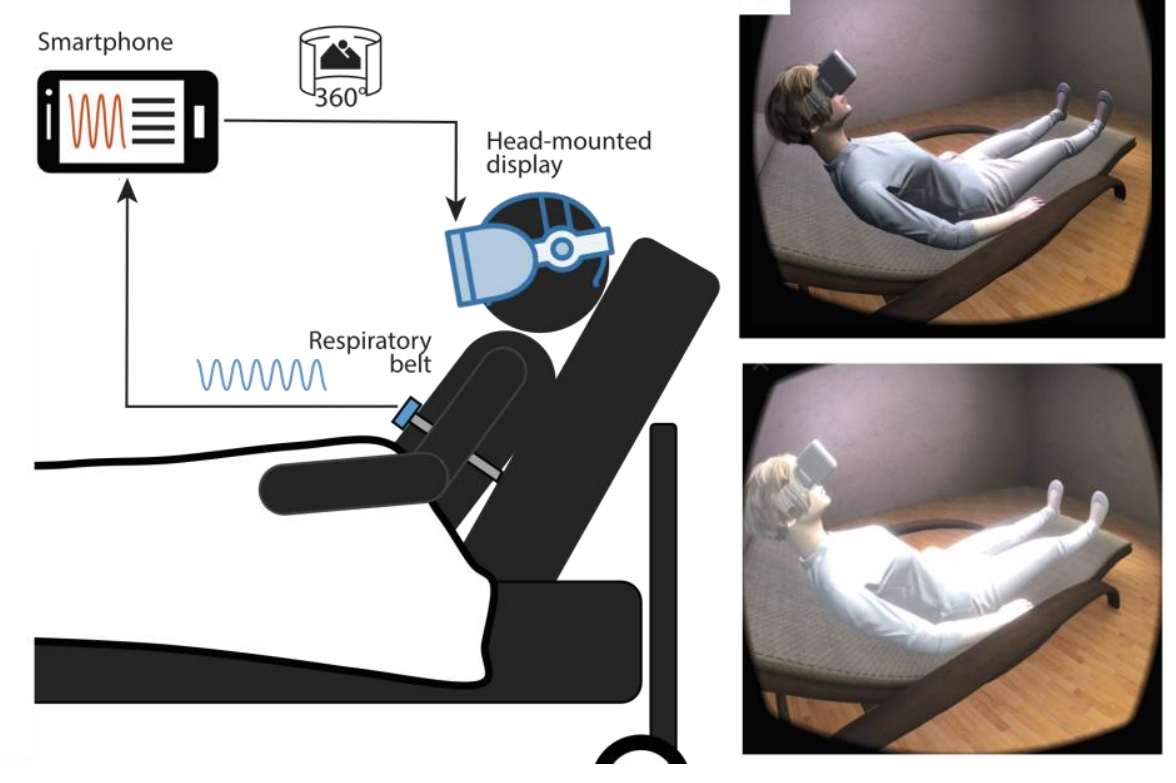

Figure 2 : Portable setup and virtual reality feedback. (A) A respiratory belt captures the respiratory movements of the chest and sends the signal to a smartphone via Bluetooth. A custom software generates the virtual environment. (B) $A$ matched-gender avatar is displayed and observed by the patients by slightly turning their head to the side. The virtual body is illuminated synchronously or asynchronously with respect to the patient's chest movements. The top image represents the end of the expiration with a low flashing intensity, while the bottom image shows the end of the inspiration corresponding to the maximal luminosity in the synchronous condition.

\section{Intervention Conditions}

In the VR environment, patients could look around and see, next to them, a gender-matching virtual body lying on a bed in a similar position as theirs (Figure 2). The virtual body was flashing in a waxing and waning visual effect. This visual effect could be in synchrony (synchronous condition) or in massive asynchrony (asynchronous condition) with the patient's respiratory movements. In the synchronous condition, the radiance of the visual flash was maximal at the end of inspiration and minimal at the end of the expiration. In the asynchronous condition, at the end of each visual flash a duration between 2.5 and 33.3 seconds is randomly generated for the next visual stimulation, such that the feedback is both phase-shifted and frequency-modulated with respect to the actual respiration.

\section{Intervention Procedure}

Once the patient was installed, a baseline assessment was performed, where patients closed their eyes (no visual feedback, NVF) and their respiratory movements recorded for two minutes. 
Participants were then asked to rate their current breathing comfort by answering two questions according to a 7 -point Likert scale (from $-3=$ Strongly disagree to $+3=$ Strongly agree): 1 ) I have difficulty breathing and 2) My breathing is enjoyable. These two items were also included in the following questionnaires and used to evaluate changes in 1) breathing discomfort, and 2) breathing comfort.

\begin{tabular}{|l|l|l|}
\hline \multicolumn{2}{|l|}{ Items } & Domain \\
\hline Q1 & $\begin{array}{l}\text { It seemed as if the flashing was my } \\
\text { respiration }\end{array}$ & $\begin{array}{l}\text { Breathing } \\
\text { awareness }\end{array}$ \\
\hline Q2 & It seemed as if I had three bodies & Control \\
\hline Q3 & $\begin{array}{l}\text { I felt as if the virtual body was breathing } \\
\text { with me }\end{array}$ & $\begin{array}{l}\text { Breathing } \\
\text { agency }\end{array}$ \\
\hline Q4 & I had difficulty breathing & Discomfort \\
\hline Q5 & $\begin{array}{l}\text { I felt as if the virtual body was drifting } \\
\text { with the flashing }\end{array}$ & Control \\
\hline Q6 & My breathing was enjoyable & Comfort \\
\hline
\end{tabular}

Table 1 Subjective questionnaire items

After baseline assessment, participants were randomly assigned in a 1:1 ratio to receive either the sequence "synchronous/asynchronous" or "asynchronous/synchronous". In each block, patients were asked to first look around in the VR room, and then to orient their gaze towards the virtual body while relaxing for 5 minutes. They were not informed that the flashing of the virtual body was related to their breathing. Each block was followed by a subjective questionnaire of six questions (7-point Likert scale) derived from previous visuo-respiratory studies ${ }^{4-6}$, (see Table 1). Question 1 evaluated the awareness of the visuo-respiratory experimental manipulation, while question 3 pertained to the breathing agency. Question 2 and 5 were proposed as control. Breathing comfort was evaluated with the same questions used in the baseline questionnaire (respectively Q4 and Q6, Table 1). Finally, to assess the acceptance and feasibility of the iVR intervention patients completed a feasibility questionnaire (7-point Likert scale, see supplementary section Table 4).

\section{Outcomes}

Two primary outcomes were proposed. One, the efficacy of the intervention was evaluated based on subjective feedback by the patients regarding their breathing comfort and discomfort. With the 
7-point Likert scale used, lower discomfort scores present a better outcome. Two, feasibility of the intervention was evaluated using a feedback questionnaire, alongside open feedback. Agreement with the questionnaire items indicates better feasibility, acceptance, and perceived outcome.

Secondary outcome measures concerned respiratory parameters as well as the embodiment of the VR feedback. Both respiratory rate (RR, breaths per minute) and respiratory rate variability (RRV, using inter-breath intervals, IBI) were measured using the respiration belt. RR and RRV were compared across the baseline and two intervention conditions. Embodiment was evaluated using a 7-point Likert scale where agreement indicates stronger embodiment. Furthermore, two control questions were included.

\section{Statistical Analysis}

Based on previous work on breathing agency ${ }^{13}$, a necessary sample size of 21 patients was estimated, using a two-sided paired t- test with an effect size of 0.65 , alpha of 0.05 and a power of 0.8 to demonstrate a difference of 0.5 between the two experimental conditions. The effect of synchrony on each measure was assessed using a linear mixed-effects model with a random intercept for each patient. In addition to the experimental condition (synchronous vs asynchronous condition), each model also included the experimental sequence (starting the experiment with synchronous or asynchronous condition) and the interaction between the experimental sequence and the experimental condition as fixed effects. The statistical significance of the interactions was assessed using the likelihood ratio test. All p-values were two-sided and statistical significance was set at a p-value of 0.05 .

Mean, standard deviation, and rating frequency (in\%) were computed for each feasibility item. In order to ensure clarity, observed percentages for ratings from 1 = Agree to $3=$ Strongly agree were grouped, indicating overall agreement with the statement. Finally, a one sided one-sample t-test was used to determine if the mean of ratings was significantly greater than zero, indicating that, at least, the majority of the patients were agreeing with the statement.

As head-mounted displays are widely used in clinical and healthy populations no specific safety analysis was performed within the scope of this study. There is no evidence that using HMDs carry risks beyond those of CRT screens (e.g., with respect to binocular vision ${ }^{18}$ or photosensitive 
epilepsy ${ }^{19}$ ). As patients remained seated during the intervention there was no risk of falling or collisions.

All analyses were performed using $R$ (version 4.1.0) and Matlab (version 2020a).

Role of the funding source

The funder of the study had no role in study design, data collection, data analysis, data interpretation, or writing of the report. 


\section{Results}

From November 2020 to April 2021, patients' enrollment, randomization and testing took place at the division of Pneumology at Geneva University Hospital. Twenty-six patients were randomly assigned either to the "asynchronous/synchronous" sequence $(n=12)$ or the "synchronous/asynchronous" sequence $(n=14)$. At the time of database lock in May 2021, data was available for all except two $(7.7 \%)$ of 26 patients (Figure 1). 


\begin{tabular}{|c|c|c|c|c|}
\hline Breathing comfort & beta & $95 \%$ CI LB & $95 \% \mathrm{CI}$ UP & P-value \\
\hline Main effect of Synchrony" & 0.542 & 0.046 & 1.037 & 0.033 \\
\hline Asynch First ${ }^{\pi}$ & 0.25 & -0.627 & 1.127 & \multirow{2}{*}{0.223} \\
\hline Synch First ${ }^{+}$ & -0.583 & -1.544 & 0.378 & \\
\hline Breathing discomfort & beta & 95\% CI LB & 95\% CI UP & P-value \\
\hline Main effect of Synchrony" & -0.5 & -1.064 & 0.064 & 0.080 \\
\hline Asynch First ${ }^{\mathbb{I}}$ & -1.333 & -2.260 & -0.407 & \multirow{2}{*}{0.221} \\
\hline Synch First $^{+}$ & -0.667 & -1.760 & 0.427 & \\
\hline Agency & beta & $95 \%$ CI LB & $95 \% \mathrm{CI}$ UP & P-value \\
\hline Main effect of Synchrony" & 1.583 & 0.335 & 2.832 & 0.014 \\
\hline Asynch First & -0.667 & -1.922 & 0.589 & \multirow{2}{*}{0.336} \\
\hline Synch First $^{+}$ & 1.167 & -1.285 & 3.618 & \\
\hline Awareness & beta & $95 \%$ CI LB & $95 \% \mathrm{CI}$ UP & P-value \\
\hline Main effect of Synchrony" & 2.167 & 1.068 & 3.266 & $<0.0001$ \\
\hline Asynch First ${ }^{\mathbb{I}}$ & 0.167 & -0.894 & 1.227 & \multirow{2}{*}{0.064} \\
\hline Synch First $^{+}$ & 2 & -0.121 & 4.121 & \\
\hline Control (Q2) & beta & $95 \%$ CI LB & $95 \% \mathrm{CI}$ UP & P-value \\
\hline Main effect of Synchrony" & 0.042 & -0.042 & 0.125 & 0.312 \\
\hline Asynch First & -3.000 & -3.135 & -2.865 & \multirow{2}{*}{0.302} \\
\hline Synch First ${ }^{+}$ & 0.083 & -0.080 & 0.246 & \\
\hline Control (Q5) & beta & $95 \%$ CI LB & $95 \%$ CI UP & P-value \\
\hline
\end{tabular}

Main effect of Synchrony ${ }^{\#}$

\begin{tabular}{|c|c|c|c|c|}
\hline Asynch First ${ }^{\pi}$ & \multicolumn{4}{|c|}{ Did not converge as data are similar in both conditions } \\
\hline Synch First ${ }^{+}$ & & & & \\
\hline Respiration Rate & beta & $95 \%$ CI LB & $95 \% \mathrm{CI}$ UP & P-value \\
\hline Main effect of Synchrony ${ }^{\#}$ & -0.275 & -1.748 & 1.198 & 0.704 \\
\hline Asynch First ${ }^{\mathbb{1}}$ & 23.346 & 19.460 & 27.231 & \\
\hline Synch First $^{+}$ & 2.685 & -0.041 & 5.410 & 0.053 \\
\hline Respiration Rate Variability & beta & $95 \%$ CI LB & $95 \% \mathrm{CI}$ UP & P-value \\
\hline Main effect of Synchrony & -0.295 & -0.779 & 0.190 & 0.222 \\
\hline Asynch First ${ }^{\mathbb{I}}$ & 4.594 & 3.344 & 5.843 & \\
\hline Synch First ${ }^{+}$ & -0.114 & -1.082 & 0.854 & \\
\hline
\end{tabular}

Table 3 Results summary for the subjective and physiological measures. \#: depicts the mean difference between synchronous and asynchronous conditions, regardless of the sequence, as well as its $\mathrm{Cl}$ estimated by the linear mixed model (the p-value corresponds to the test of this difference being equal to zero); $\mathfrak{q}$ : depicts the mean difference between synchronous and asynchronous conditions and its $\mathrm{Cl}$ estimated by the linear mixed model for the experimental sequence "Asynchronous first" (the p-value corresponds to the result of the interaction test); +: depicts the mean difference between synchronous and asynchronous conditions and its $\mathrm{Cl}$ estimated by the linear mixed model for the experimental sequence "Synchronous first" (the p-value corresponds to the result of the interaction test). Cl: confidence interval, LB: Lower Bound, UB: Upper Bound. Subjective ratings were measured using a 7-point Likert scale with $-3=$ Strongly disagree, -2 = Disagree; $-1=$ Somewhat disagree; $0=$ Neither agree nor disagree; 1 = Somewhat agree; $2=$ Agree; $3=$ Strongly agree. 
The main outcome measures of this study were breathing comfort ratings. The mean comfort rating was $0.75 \pm 1.78$ during the asynchronous condition and $1.29 \pm 1.37$ during the synchronous condition, with an estimated difference of $0.54(95 \% \mathrm{Cl} 0.05$ to 1.04$)(p<0.05)$ between conditions (Figure 3.A). The order of conditions did not impact these findings (no significant interaction for comfort rating between experimental conditions (asynchronous or synchronous) and experimental sequence (starting the experiment with asynchronous stimulations or starting it with synchronous stimulations)). Moreover, post-hoc paired one-sided t-tests, confirmed a significant difference between breathing comfort ratings during the experimental (synchronous) condition compared to baseline. Such difference was absent for the control (asynchronous) condition, excluding a mere effect of VR distraction (see supplementary section for statistical details). For the assessment of discomfort, even though a similar trend was observed in the data, neither the main effect of experimental condition nor its interaction with the experimental sequence reached significance.

The secondary outcome measures of this study were the embodiment-related subjective ratings and the physiological measures. The mean agency rating was $-0.67 \pm 2.26$ during the asynchronous condition and $0.92 \pm 2.26$ during the synchronous condition, with an estimated difference of 1.58 (95\% Cl 0.34 to 2.83) ( $p<0.05$ ) between conditions (Figure 3.B). The order of conditions did not impact agency ratings (no interaction between experimental conditions and experimental sequence). The mean awareness rating was $-0.67 \pm 2.14$ during the asynchronous condition and 1.5 1.8 during the synchronous condition, with an estimated difference of 2.17 (95\% Cl 1.07 to 3.27 ) $(p<0.0001$ ) between conditions (Figure 3.C) and the order of conditions did not impact awareness ratings. For the first control item, neither the main effect of experimental condition nor its interaction with the experimental sequence reached significance. For the second control item, ratings did not differ between the two experimental condition $(-2.79 \pm 0.51)$, leading to models not converging. For both $R R$ and its variability, neither the main effects of experimental condition nor its interactions with the experimental sequence reached significance; suggesting that the effect of the intervention on comfort rating was not related to respiratory signal modulations. 
A

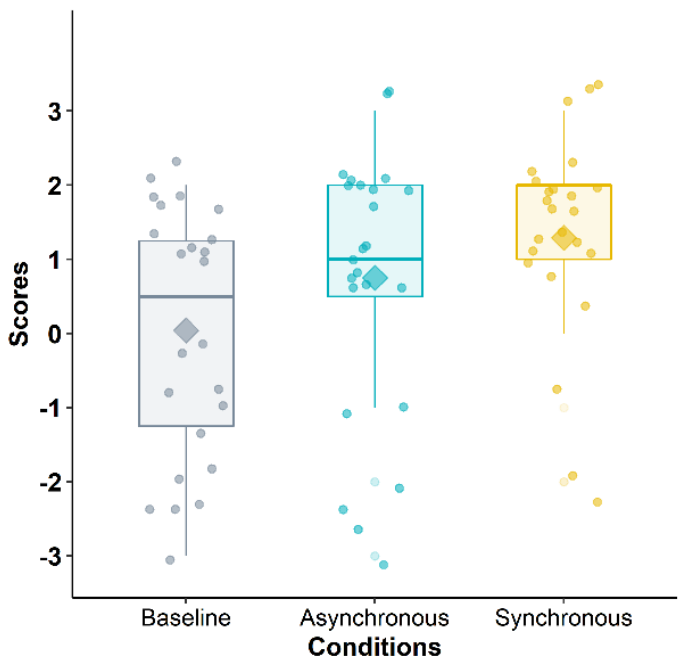

B

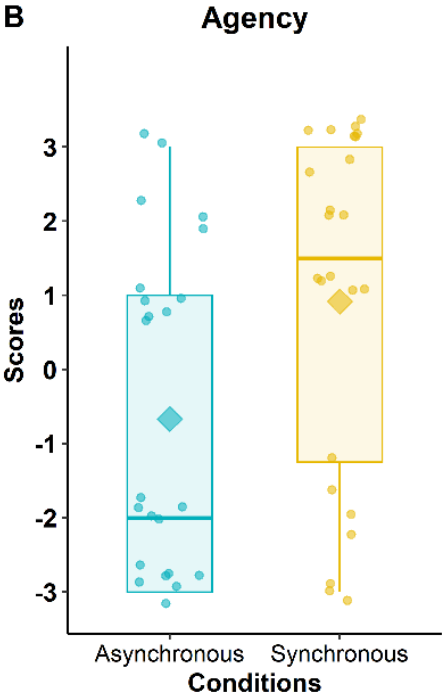

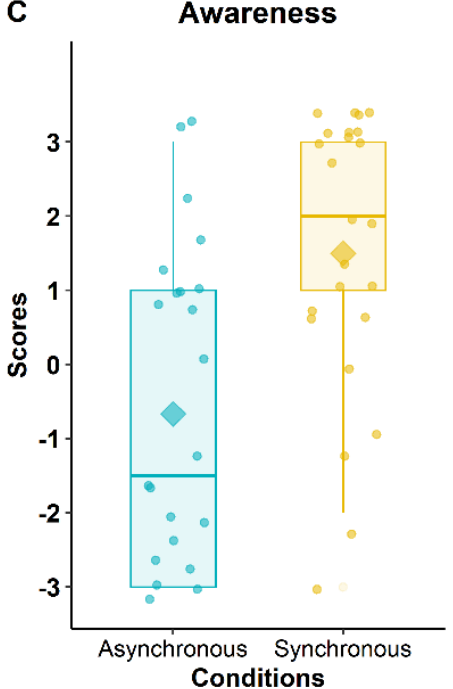

Figure 3 Breathing comfort ( A), Agency (B) and Awareness (C) test results. The boxplots depicting subjects' ratings during asynchronous condition compared to the synchronous condition, independent of experimental sequence. The thick line within a box plot represents the median, the diamond represents the mean, the upper boundary of the box indicates the 25th percentile and lower boundary the 75th percentile. The whiskers above and below the box indicate the minimal and maximal values, while points above the upper or below the whiskers indicate outliers. Subjective ratings were measured using a 7-point Likert scale with $-3=$ Strongly disagree, $-2=$ Disagree; $-1=$ Somewhat disagree; 0 = Neither agree nor disagree; 1 = Somewhat agree; 2 = Agree; $3=$ Strongly agree .

The evaluation of the iVR intervention feasibility was the second main outcome measure of this study. As depicted on Figure 4 \& Figure 5, 91.2\% of the patients were satisfied by the intervention (Satisfaction: mean score $=1.75 \pm 1.65 ; t=5.20, p<0.0001,95 \% \mathrm{Cl} 1.17$ to inf ). In addition, $66.7 \%$ rated the iVR intervention as beneficial for their breathing (Respiratory benefit: $0.71 \pm 1.92$, $t=1.81, p<0.05,95 \% \mathrm{Cl} 0.04$ to inf $) .50 \%$ reported that the VR intervention made them feel better (Well-being benefit: $0.17 \pm 2.3, t=0.36, p>0.05,95 \% \mathrm{Cl}-0.64$ to inf). Also, $45.8 \%$ of the sample would like to continue using the device during their recovery (Rehabilitation: $0.04 \pm 2.03$, $\mathrm{t}=0.10, \mathrm{p}>0.05,95 \% \mathrm{Cl}-0.67$ to inf) and when at home (Home use: $-0.34 \pm 2.22, \mathrm{t}=-0.74, \mathrm{p}>$ $0.05,95 \% \mathrm{Cl}-1.11$ to inf). Finally, $37.5 \%$ of the sample would have liked to use the intervention earlier during their stay at the hospital (Hospital use: $-0.083 \pm 1.86, \mathrm{t}=-0.22, \mathrm{p}>0.05,95 \% \mathrm{Cl}$ 0.74 to inf). Descriptive statistics and statistical tests are described in the supplementary section (Table 6). 


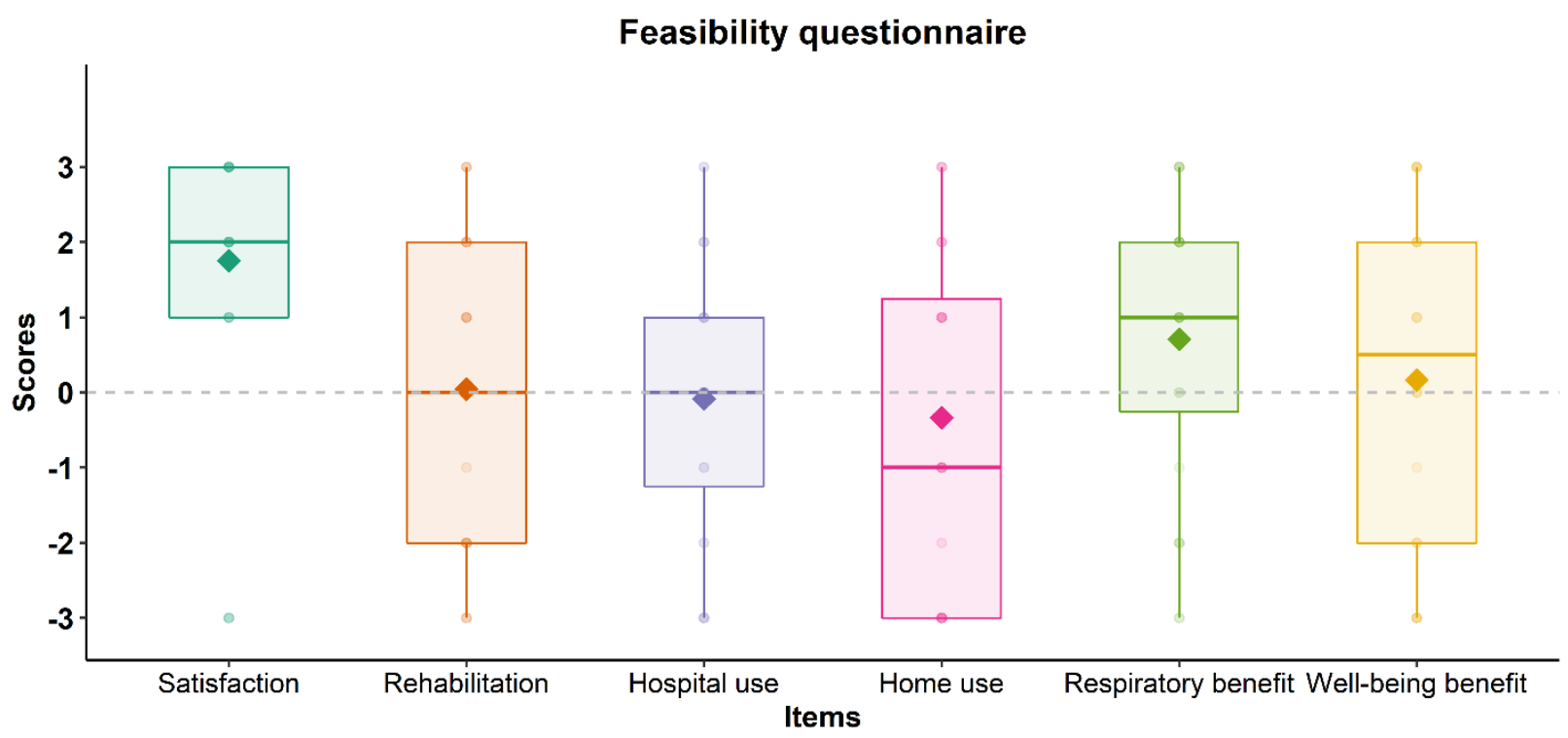

Figure 4 Feasibility scores for all items. The boxplots depicting subjects' ratings for feasibility items. The thick line within a box plot represents the median, the diamond represents the mean, the upper boundary of the box indicates the 25th percentile and lower boundary the 75th percentile. The whiskers above and below the box indicate the minimal and maximal values, while points above the upper or below the whiskers indicate outliers. Subjective ratings were measured using a 7-point Likert scale with $-3=$ Strongly disagree, $-2=$ Disagree; $-1=$ Somewhat disagree; $0=$ Neither agree nor disagree; 1 = Somewhat agree; 2 = Agree; $3=$ Strongly agree. 

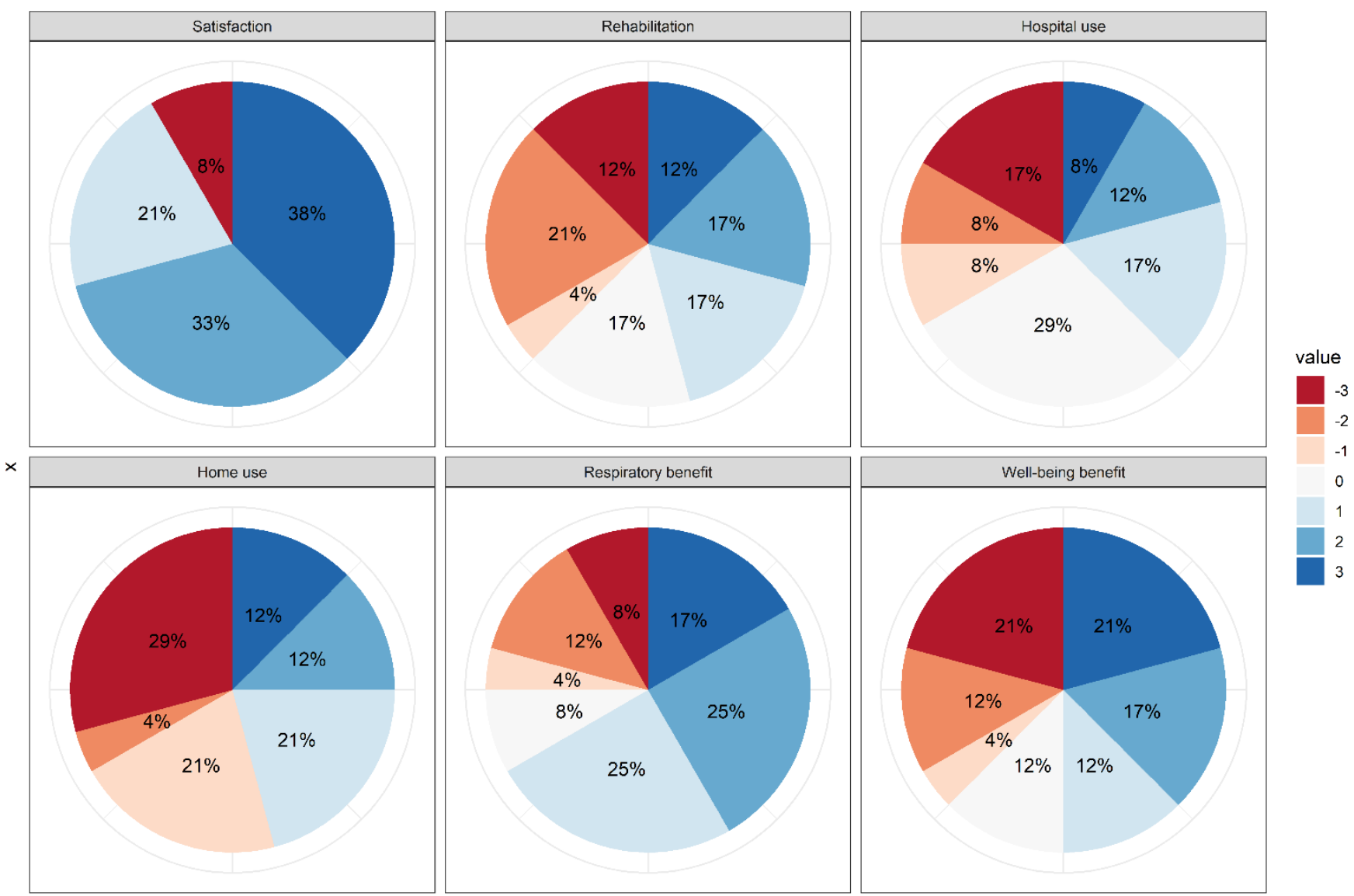

Figure 5 Percentage of feasibility scores for all items, rated on a 7-point Likert scale, with $-3=$ Strongly disagree, -2 = Disagree; -1 = Somewhat disagree; 0 = Neither agree nor disagree; 1 = Somewhat agree; 2 = Agree; $3=$ Strongly agree 


\section{Panel: Research in context}

\section{Evidence before the study}

We searched MEDLINE (PubMed) to identify relevant studies investigating the relationships between respiration, refractory breathlessness/dyspnea, virtual reality and COVID-19 infection. We also explored studies/randomized controlled trials (RCTs) of VR-based immersive DTx aimed at alleviating pain/refractory breathlessness. To maximize sensitivity, we also searched for citations in Google Scholar, Scopus, and Web of Science. Search terms included "COVID-19", "coronavirus", "breathlessness", "dyspnea", "virtual reality", "breathing", "respiration", "rehabilitation", "pain" and "immersive digiceuticals", either separately or in combination. In previous work in healthy populations, the manipulation of visuo-respiratory signals using VR was related to increased feeling of breathing control, reduced negative emotion during induceddyspnea and changes in physiological measures of breathing signal. Breathlessness is poorly associated to physiological impairment measured by pulmonary function tests or lung imaging in long COVID-19, which also implies that the brain could be a possible target for a neurorehabilitation. Alleviating breathlessness in Long COVID patients requires a clinically proven, easily adaptable, and inexpensive intervention.

\section{Added value of this study}

Previous iVR interventions manipulating visual cardiac feedback led to pain alleviation in clinical populations such as complex regional pain syndrome or spinal cord injury. In the respiratory domain, the manipulation of visuo-respiratory stimulations has been associated with reduced negative emotional state related to breathlessness, with changes in respiratory signals (rate, tidal volume variability and respiration amplitude), and with increased breathing agency (i.e. the feeling of sensing one's breathing command). In our COVVR controlled, randomized, single-blind crossover clinical study of 26 patients presenting with refractory breathlessness after COVID-19 infection, the effect of visuo-respiratory stimulations improved breathing comfort. Most patients were satisfied by the intervention and rated the iVR intervention as beneficial to improve this debilitating symptom. 


\section{Implications of all the available evidence}

Given the scarcity of evidence-based treatment to treat refractory breathlessness, and the benefit of visuo-respiratory iVR-based intervention in patients recovering from COVID-19, we propose that our iVR-based immersive DTx is a safe and inexpensive neuro-rehabilitation tool that could be considered for use to increase breathing comfort in patients. Replication of our results in other clinical groups suffering from refractory breathlessness is recommended. 


\section{Discussion}

In COVVR, an iVR-based immersive DTx improved breathing comfort in patients with RB recovering from severe COVID-19 pneumonia. During synchronous visuo-respiratory stimulation, breathing comfort significantly improved compared to the asynchronous control condition and to a baseline condition. A similar effect of the intervention was observed for breathing agency, i.e. the feeling of being in control of one's breathing. Finally, the vast majority of the sample reported a global satisfaction regarding the VR intervention and, more importantly, indicated that the iVR feedback improved their breathing. Our findings were neither modulated by the experimental sequence, nor can they be explained by VR distraction, or by changes in the respiratory measures during the intervention.

$\mathrm{RB}$, a common but underreported condition, is defined as breathlessness that persists despite optimal treatment of the underlying pathophysiology. This results in important disabilities impacting cognition, locomotion and mental health ${ }^{3,4,20}$. Mounting evidence using functional neuroimaging suggests that patients suffering from RB may exhibit brain "hypersensitivity" to afferent respiratory signals as a result of learned expectations during daily physical activities. ${ }^{20}$ Perception and anticipation of breathlessness are known to share neural correlates such as brainstem areas or the insular cortex ${ }^{21-23}$. It is likely that our manipulation interacts with such systems. Consequently, once treatment of the underlying pathophysiology has been optimized, the brain may become a potential target for pharmacological and non-pharmacological interventions. Pharmacological treatments may also be useful; low dose oral sustained-release morphine administered for $\mathrm{RB}$ is associated with improved health status in COPD without affecting $\mathrm{PaCO} 2$ or causing serious side-effects, especially in patients with mMRC stage 3 to $4^{20}$. Pulmonary rehabilitation, an evidence-based multidisciplinary non-pharmacological intervention has also been shown to modify neural responses to learned breathlessness associations, which corresponds from a clinical point of view to central desensitization to dyspnea as an improvement in lung mechanics is most often not observed with this intervention ${ }^{21}$.

Using iVR, the impact of visuo-respiratory stimulations on breathing has already been documented in healthy subjects, where the exposure to visuo-respiratory feedback led to increased breathing agency ${ }^{12}$, and to changes in respiratory rate ${ }^{25}$, tidal volume variability ${ }^{14}$, and respiration amplitude ${ }^{26}$. Moreover, a greater positive emotional state, during experimentally induced dyspnea (loaded breathing), has also been observed during a similar iVR intervention ${ }^{13}$. 16 
iVR DTx are becoming popular in the field of chronic pain management as standard therapies have many shortcomings such as the prescription of controversial opioid medication ${ }^{25}$. Of note, breathlessness and pain share striking similarities ${ }^{6}$. They engage similar brain networks ${ }^{6}$, are best characterized by multidimensional models ${ }^{28}$ and both respond to opioid treatment. As the global COVID-19 pandemic has progressed, a significant proportion of patients experience prolonged symptoms beyond the initial period of acute infection, such as RB. Such clinical cases are known as "Long COVID"15. The increasing number of patients isolated for prolonged periods has stressed an urgent need to develop multidisciplinary rehabilitation strategies that can be individualized and adapted to accommodate patients' needs in intensive care or at home ${ }^{24}$. Given the COVVR findings, we propose that our iVR DTx is a feasible and safe neuro-rehabilitation tool that could be considered for use to improve breathing comfort in patients experiencing refractory breathlessness after COVID-19 infection. Of note, while our intervention has been tested in patients recovering from COVID-19, its use could be extended to numerous respiratory conditions characterized by refractory breathlessness, as iVR DTx intervention directly impacts central processing of respiratory signals to alleviate breathlessness.

Our study comes with certain limitations. The main limitation is the small sample size, although it has been based on an adequate power calculation for a proof-of-concept study. Another limitation is that we included only a homogeneous population of patients recovering from severe COVID19 infection. Our intervention should now be tested in a larger cohort of patients with refractory breathlessness to improve generalizability. Another important unanswered question is whether central desensitization to breathlessness while training with iVR DTx can persist when patients are off-treatment. Longer term studies with breathlessness ecological momentary assessments are therefore needed before such treatment can be widely accepted in a clinical setting.

One important innovation this study demonstrates is the improved efficacy linked to real-time biofeedback, going beyond the classically admitted distraction from suffering offered by iVR ${ }^{30}$. Relying on the recent expansion of Internet of Things (IOT) technologies and of wearable physiological sensors in particular, our approach highlights how iVR DTx interventions can now more specifically target the neural mechanisms of pain and RB. Although in its current form our technology did not fulfill the expectations for home-use in our patients' cohort, it paves the way 
for future e-health products that would not only provide monitoring but also a continuum of interventions from hospital bed to patient's home.

In conclusion, our study shows that an immersive VR-based digital therapeutic can improve breathing comfort in patients recovering from severe COVID-19 pneumonia. Global satisfaction and respiratory benefit from the patients are reported, attesting to the feasibility of this simple intervention. Although more clinical studies are needed, our iVR-based DTx may become a key factor of multi-dimensional treatment of refractory breathlessness. 


\section{Contribution}

Every author named at the start of the article contributed to the study: Conceptualization ( $A D, B O$, $B S, H B, K O, S T$ ), Data curation (BS, FJ), Formal Analysis (FJ, BS), Funding acquisition (AD, BO, $B S$ ), Investigation (FJ, SA), Methodology (AD, BO, BS, FJ, HB, KO), Project administration (AD, FJ, SA), Resources, Software (CS, HB, LF), Supervision (AD, BO, BS, KO), Visualization (BS, FJ), Writing - original draft (BS, FJ), Writing - review \& editing (AD, BO, BS, CS, HB, KO, ST).

All authors confirm that they had full access to all the data in the study and accept responsibility to submit for publication. FJ and BS verified the data.

\section{Declaration of interests}

We declare no competing interests.

\section{Funding}

Dr Betka Sophie's salary has been funded by a Marie Skłodowska-Curie Individual Fellowship (H2020-MSCA-IF-2019 894111/ RESPVR), awarded by the European Commission. The project has been partly funded by the Bertarelli Foundation.

\section{Acknowledgements}

We thank Dr Elise Dupuis-Lozeron and Dr Fosco Bernasconi for their statistical advice. 


\section{References $(\max 30)$}

1. Johnson, M. J. et al. Towards an expert consensus to delineate a clinical syndrome of chronic breathlessness. Eur. Respir. J. 49, 1602277 (2017).

2. Morélot-Panzini, C. et al. Breathlessness despite optimal pathophysiological treatment: on the relevance of being chronic. Eur. Respir. J. 50, (2017).

3. Lawi, D. et al. Experimental dyspnoea interferes with locomotion and cognition: a randomised trial. Eur. Respir. J. 56, (2020).

4. Janssen, D. J. A., Wouters, E. F. M. \& Spruit, M. A. Psychosocial consequences of living with breathlessness due to advanced disease. Curr. Opin. Support. Palliat. Care 9, 232-237 (2015).

5. Stevens, J. P. et al. Association of dyspnoea, mortality and resource use in hospitalised patients. Eur. Respir. J. 58, 1902107 (2021).

6. von Leupoldt, A. et al. Dyspnea and pain share emotion-related brain network. Neurolmage 48, 200-206 (2009).

7. Betka, S., Adler, D., Similowski, T. \& Blanke, O. Breathing control, brain, and bodily selfconsciousness: toward immersive digiceuticals to alleviate respiratory suffering. (under review).

8. Park, H. D. \& Blanke, O. Coupling Inner and Outer Body for Self-Consciousness. Trends Cogn. Sci. 23, 377-388 (2019).

9. Rognini, G. \& Blanke, O. Cognetics: Robotic Interfaces for the Conscious Mind. Trends Cogn. Sci. 20, 162-164 (2016).

10. Solcà, M. et al. Enhancing analgesic spinal cord stimulation for chronic pain with personalized immersive virtual reality. Pain (2020) doi:10.1097/j.pain.0000000000002160. 
11. Pozeg, P. et al. Virtual reality improves embodiment and neuropathic pain caused by spinal cord injury. Neurology 89, 1894-1903 (2017).

12. Adler, D., Herbelin, B., Similowski, T. \& Blanke, O. Breathing and sense of self: visuorespiratory conflicts alter body self-consciousness. Respir. Physiol. Neurobiol. 203, 68-74 (2014).

13. Allard, E. et al. Interferences between breathing, experimental dyspnoea and bodily selfconsciousness. Sci. Rep. 7, 9990 (2017).

14. Betka, S. et al. Mechanisms of the breathing contribution to bodily self-consciousness in healthy humans: Lessons from machine-assisted breathing? Psychophysiology e13564 (2020) doi:10.1111/psyp.13564.

15. Michelen, M. et al. Characterising long term Covid-19: a living systematic review. 2020.12.08.20246025 https://www.medrxiv.org/content/10.1101/2020.12.08.20246025v2 (2021) doi:10.1101/2020.12.08.20246025.

16. Lerum, T. V. et al. Dyspnoea, lung function and CT findings three months after hospital admission for COVID-19. Eur. Respir. J. (2020) doi:10.1183/13993003.03448-2020.

17. Spruit, M. A. et al. COVID-19: Interim Guidance on Rehabilitation in the Hospital and PostHospital Phase from a European Respiratory Society and American Thoracic Societycoordinated International Task Force. Eur. Respir. J. (2020) doi:10.1183/13993003.021972020.

18. Peli, E. The visual effects of head-mounted display (HMD) are not distinguishable from those of desk-top computer display. Vision Res. 38, 2053-2066 (1998).

19. Tychsen, L. \& Thio, L. L. Concern of Photosensitive Seizures Evoked by 3D Video Displays or Virtual Reality Headsets in Children: Current Perspective. Eye Brain 12, 45-48 (2020). 
20. Verberkt, C. A. et al. Effect of Sustained-Release Morphine for Refractory Breathlessness in Chronic Obstructive Pulmonary Disease on Health Status: A Randomized Clinical Trial. JAMA Intern. Med. 180, 1306-1314 (2020).

21. Marlow, L. L., Faull, O. K., Finnegan, S. L. \& Pattinson, K. T. S. Breathlessness and the brain: the role of expectation. Curr. Opin. Support. Palliat. Care 13, 200-210 (2019).

22. Vlemincx, E., Sprenger, C. \& Büchel, C. Expectation and dyspnea: The neurobiological basis of respiratory nocebo effects. Eur. Respir. J. (2021) doi:10.1183/13993003.03008-2020.

23. Harrison, O. K. et al. Interoception of breathing and its relationship with anxiety. bioRxiv 2021.03.24.436881 (2021) doi:10.1101/2021.03.24.436881 .

24. Herigstad, M. et al. Treating breathlessness via the brain: changes in brain activity over a course of pulmonary rehabilitation. Eur. Respir. J. 50, (2017).

25. Czub, M. \& Kowal, M. Respiration Entrainment in Virtual Reality by Using a Breathing Avatar. Cyberpsychology Behav. Soc. Netw. 22, 494-499 (2019).

26. Monti, A., Porciello, G., Tieri, G. \& Aglioti, S. M. The 'embreathment' illusion highlights the role of breathing in corporeal awareness. J. Neurophysiol. 123, 420-427 (2020).

27. Trost, Z., France, C., Anam, M. \& Shum, C. Virtual reality approaches to pain: toward a state of the science. PAIN 162, 325-331 (2021).

28. Lansing, R. W., Gracely, R. H. \& Banzett, R. B. The multiple dimensions of dyspnea: review and hypotheses. Respir. Physiol. Neurobiol. 167, 53-60 (2009).

29. Venkatesan, P. NICE guideline on long COVID. Lancet Respir. Med. 9, 129 (2021).

30. Spiegel, B. et al. Virtual reality for management of pain in hospitalized patients: A randomized comparative effectiveness trial. PLOS ONE 14, e0219115 (2019). 


\section{Supplementary}

Supplementary material

\begin{tabular}{|l|l|l|}
\hline \multicolumn{2}{|l|}{ Items } & Domain \\
\hline Q1 & Did you enjoy the VR experience? & Satisfaction \\
\hline Q2 & $\begin{array}{l}\text { Would you like to continue using the device during your } \\
\text { recovery? }\end{array}$ & Rehabilitation \\
\hline Q3 & $\begin{array}{l}\text { Would you have liked to use this earlier during your stay at } \\
\text { the hospital? }\end{array}$ & Hospital Use \\
\hline Q4 & Would you like to continue using the device at home? & Home Use \\
\hline Q5 & Do you think the VR feedback improved your breathing? & Respiratory benefit \\
\hline Q6 & Did the VR feedback make you feel better? & Well-being benefit \\
\hline
\end{tabular}

Table 4 Feasibility questionnaire 


\section{Supplementary Results}

Means and standard deviations in function of the experimental sequence

\begin{tabular}{|l|c|c|c|c|c|c|c|c|}
\hline & \multicolumn{4}{|c|}{ Asynchronous Condition } & \multicolumn{3}{c|}{ Synchronous Condition } \\
\cline { 2 - 9 } & $\begin{array}{l}\text { Asynchronous } \\
\text { first (n=12) }\end{array}$ & \multicolumn{2}{c|}{$\begin{array}{c}\text { Synchronous } \\
\text { first (n=12) }\end{array}$} & \multicolumn{2}{c|}{$\begin{array}{c}\text { Asynchronous } \\
\text { first (n=12) }\end{array}$} & \multicolumn{2}{c|}{$\begin{array}{c}\text { Synchronous } \\
\text { first (n=12) }\end{array}$} \\
\hline Measures & Mean & $S D$ & Mean & $S D$ & Mean & $S D$ & Mean & $S D$ \\
\hline Comfort & 0.250 & 1.765 & 1.250 & 1.712 & 1.083 & 1.443 & 1.500 & 1.314 \\
\hline Discomfort & -1.333 & 1.723 & -0.667 & 1.670 & -1.500 & 1.784 & -1.500 & 1.446 \\
\hline Agency & -0.667 & 2.27 & -0.667 & 2.348 & 0.333 & 2.674 & 1.5 & 1.679 \\
\hline Awareness & 0.167 & 2.167 & -1.5 & 1.834 & 1.333 & 2.188 & 1.667 & 1.371 \\
\hline Control (Q2) & -3.000 & 0.000 & -2.917 & 0.289 & -3.000 & 0.000 & -2.833 & 0.389 \\
\hline Control (Q5) & -2.833 & 0.389 & -2.750 & 0.622 & -2.833 & 0.389 & -2.750 & 0.622 \\
\hline Respiratory rate & 23.346 & 6.242 & 21.750 & 5.746 & 21.728 & 8.738 & 22.817 & 6.564 \\
\hline $\begin{array}{l}\text { Respiratory rate } \\
\text { Variability }\end{array}$ & 4.594 & 2.711 & 4.127 & 1.912 & 4.356 & 2.422 & 3.775 & 1.724 \\
\hline
\end{tabular}

Table 5 Means and standard deviations for the asynchronous and asynchronous conditions, in function of the experimental sequence.

\section{Breathing comfort - Tests against baseline}

Using post-hoc paired one-sided t-tests, we found a significant difference between breathing comfort ratings during the synchronous condition compared to baseline (Difference: $1.25 \pm 0.431$, $\mathrm{t}=2.901, \mathrm{p}<0.01,95 \% \mathrm{Cl} 0.511$ to inf). This was not observed between breathing comfort ratings during the asynchronous condition compared to baseline (Difference: $0.708 \pm 0.547, t=1.296, p>$ $0.05,95 \% \mathrm{Cl}-0.229$ to inf), excluding a mere effect of VR distraction. 
Additional figures

A

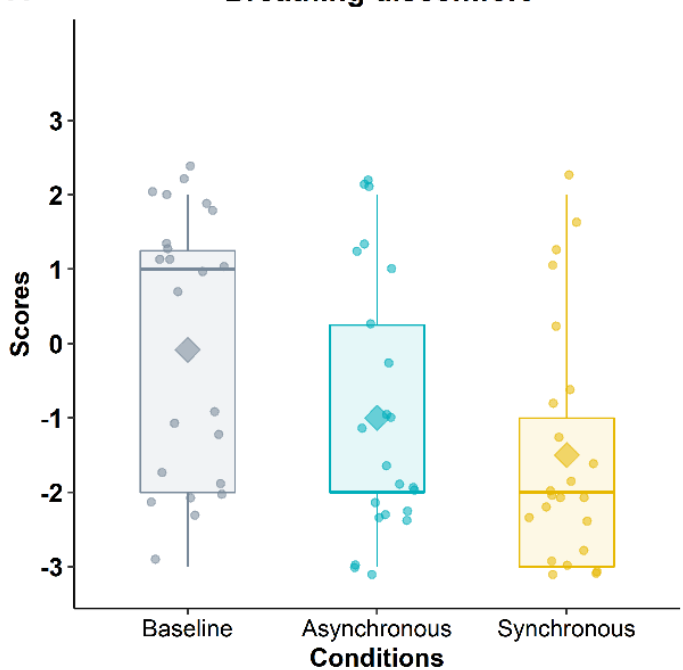

B

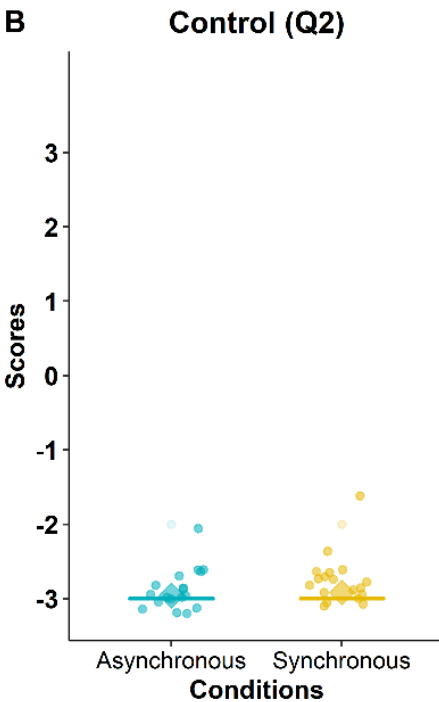

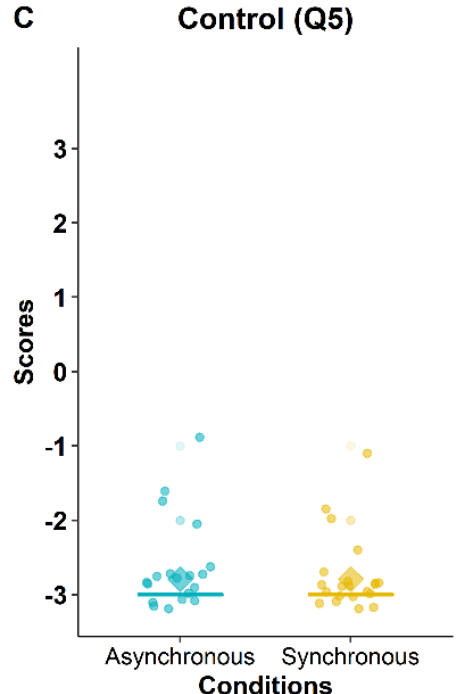

Figure 6 Subjective measures for which the main effect of the experimental manipulation was not significant. The boxplots depicting subjects' ratings during asynchronous condition compared to the synchronous condition, independent of experimental sequence. The thick line within a box plot represents the median, the diamond represents the mean, the upper boundary of the box indicates the 25th percentile and lower boundary the 75th percentile. The whiskers above and below the box indicate the minimal and maximal values, while points above the upper or below the whiskers indicate outliers. Subjective ratings were measured using a 7 -point Likert scale with $-3=$ Strongly disagree, 2 = Disagree; $-1=$ Somewhat disagree; $0=$ Neither agree nor disagree; $1=$ Somewhat agree; 2 = Agree; $3=$ Strongly agree. Discomfort item:"I had difficulty breathing" 
A

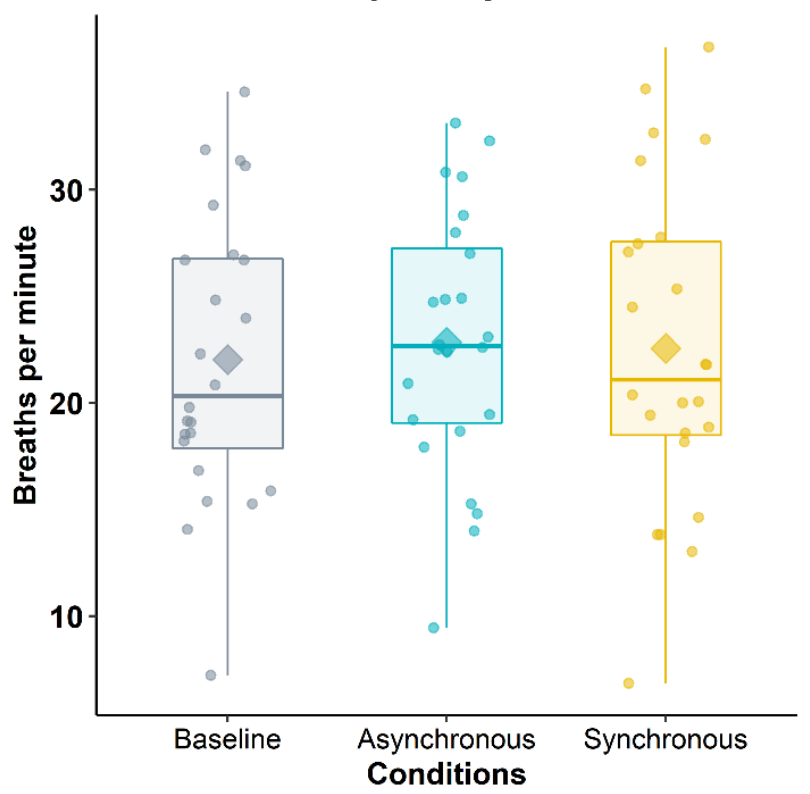

B Respiratory Rate Variability

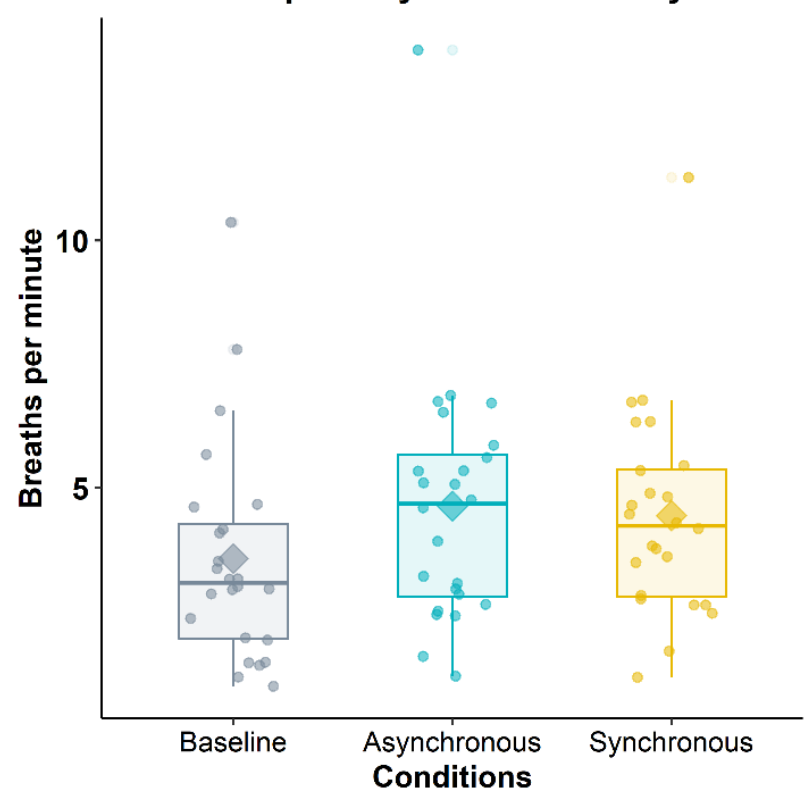

Figure 7 Objective measures for which the main effect of the experimental manipulation was not significant. The boxplots depicting subjects' physiology signal during asynchronous condition compared to the synchronous condition, independent of experimental sequence. The thick line within a box plot represents the median, the diamond represents the mean, the upper boundary of the box indicates the 25th percentile and lower boundary the 75th percentile. The whiskers above and below the box indicate the minimal and maximal values, while points above the upper or below the whiskers indicate outliers. Subjective ratings were measured using a 7-point Likert scale with $-3=$ Strongly disagree, 2 = Disagree; -1 = Somewhat disagree; 0 = Neither agree nor disagree; 1 = Somewhat agree; $2=$ Agree; $3=$ Strongly agree. 
Feasibility

\begin{tabular}{|c|c|c|c|c|c|l|l|}
\hline Items & Mean & $\mathbf{s d}$ & $\mathbf{t}$ & $\mathbf{d f}$ & $\mathbf{p}$-value & $\mathbf{C I}$ LB & CI UB \\
\hline Satisfaction & 1.75 & 1.649 & 5.201 & 23.000 & 0.000 & 1.173 & inf \\
\hline Rehabilitation & 0.042 & 2.032 & 0.100 & 23.000 & 0.460 & -0.669 & inf \\
\hline Usage at the hospital & -0.083 & 1.863 & -0.219 & 23.000 & 0.586 & -0.735 & inf \\
\hline Usage at home & -0.333 & 2.22 & -0.736 & 23.000 & 0.765 & -1.110 & inf \\
\hline Respiratory benefit & 0.708 & 1.922 & 1.806 & 23.000 & 0.042 & 0.036 & inf \\
\hline Well-being benefit & 0.167 & 2.297 & 0.355 & 23.000 & 0.363 & -0.637 & inf \\
\hline
\end{tabular}

Table 6 Descriptive statistics and statistical tests of the feasibility items. Ratings were measured using a 7-point Likert scale with $-3=$ Strongly disagree, $-2=$ Disagree; $-1=$ Somewhat disagree; $0=$ Neither agree nor disagree; $1=$ Somewhat agree; 2 = Agree; $3=$ Strongly agree. $\mathrm{N}$ =number of observations, $\mathrm{SD}=$ standard deviation, $\mathrm{df}=$ degree of 